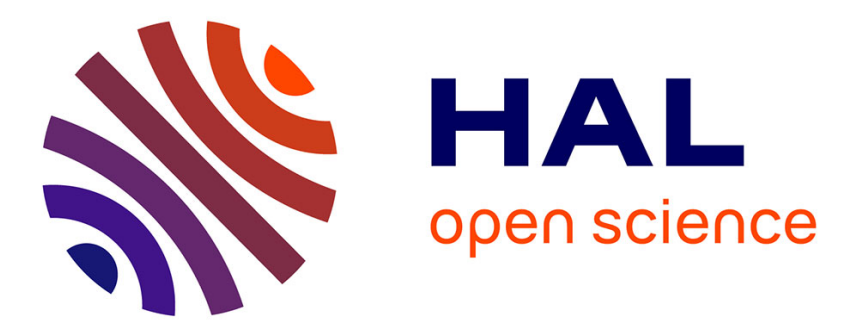

\title{
The Capacitated Vehicle Routing Problem with Evidential Demands: a Belief-Constrained Programming Approach
}

Nathalie Helal, Frédéric Pichon, Daniel Cosmin Porumbel, David Mercier, Éric Lefèvre

\section{To cite this version:}

Nathalie Helal, Frédéric Pichon, Daniel Cosmin Porumbel, David Mercier, Éric Lefèvre. The Capacitated Vehicle Routing Problem with Evidential Demands: a Belief-Constrained Programming Approach. BELIEF'2016, Sep 2016, Prague, Czech Republic. pp.212-221, 10.1007/978-3-319-455594_22. hal-03337226

\author{
HAL Id: hal-03337226 \\ https://hal.science/hal-03337226
}

Submitted on 7 Sep 2021

HAL is a multi-disciplinary open access archive for the deposit and dissemination of scientific research documents, whether they are published or not. The documents may come from teaching and research institutions in France or abroad, or from public or private research centers.
L'archive ouverte pluridisciplinaire HAL, est destinée au dépôt et à la diffusion de documents scientifiques de niveau recherche, publiés ou non, émanant des établissements d'enseignement et de recherche français ou étrangers, des laboratoires publics ou privés. 


\title{
The Capacitated Vehicle Routing Problem with Evidential Demands: a Belief-Constrained Programming Approach
}

\author{
Nathalie Helal ${ }^{1}$, Frédéric Pichon ${ }^{1}$, Daniel Porumbel $^{2}$, David Mercier ${ }^{1}$ and Éric \\ Lefèvre $^{1}$ \\ ${ }^{1}$ Univ. Artois, EA 3926, Laboratoire de Génie Informatique et d'Automatique de \\ l'Artois (LGI2A), F-62400 Béthune, France. \\ \{nathalie_helal@ens.univ-artois.fr,firstname.lastname@univ-artois.fr\} \\ ${ }^{2}$ Conservatoire National des Arts et Métiers, EA 4629, Cedric, 75003 Paris, France. \\ daniel.porumbel@cnam.fr
}

\begin{abstract}
This paper studies a vehicle routing problem, where vehicles have a limited capacity and customer demands are uncertain and represented by belief functions. More specifically, this problem is formalized using a belief function based extension of the chance-constrained programming approach, which is a classical modeling of stochastic mathematical programs. In addition, it is shown how the optimal solution cost is influenced by some important parameters involved in the model. Finally, some instances of this difficult problem are solved using a simulated annealing metaheuristic, demonstrating the feasibility of the approach.
\end{abstract}

Keywords: Vehicle routing problem, Stochastic programming, Chanceconstrained programming, Belief functions.

\section{Introduction}

The Capacitated Vehicle Routing Problem with Stochastic Demands (CVRPSD) is a Vehicle Routing Problem (VRP) that asks to determine the set of routes of minimum cost that can serve a set of customers with stochastic demands, while respecting the capacity limit of each vehicle 8. This stochastic integer linear program can be modeled via the Chance-Constrained Programming (CCP) approach [26, which is one of the main approaches to addressing stochastic mathematical programs. Modeling the CVRPSD via CCP amounts to having a constraint, which states that the probability that any route exceeds vehicle capacity, must be below a given (small) value.

Belief function theory [14 is an alternative uncertainty framework to probability theory. Uncertainty on customer demands may be naturally represented by belief functions in various situations; for instance, when sources providing pieces of information on customer demands, are assumed to be partially reliable or biased 12. In such case, a new VRP is obtained, which may be called the Capacitated VRP with Evidential Demands (CVRPED), where evidential means that uncertainty on customer demands is modeled by belief functions. 
Few papers 10 159 have been dedicated to handling uncertainty within optimization problems using belief functions. These papers only addressed the case of continuous linear programs, which are usually far more easier to solve than their discrete counterparts. Most notably, Masri and Ben Abdelaziz 9] generalized the CCP approach to linear programs involving uncertainty represented by belief functions, which they coined the Belief-Constrained Programming (BCP) approach to the belief linear programming problem.

In this paper, we study the extension of the CCP approach to an integer linear program involving uncertainty represented by belief functions, which is the CVRPED, leading to what may be called the BCP approach to the CVRPED. Being a derivative from the large class of VRP, which are NP-hard and may be tackled using metaheuristics [3], we adapt a simulated annealing metaheuristic [7] to find solutions to the CVRPED modeled via the BCP approach.

This paper is structured as follows. Section 2 contains a brief reminder of CVRPSD modeled via CCP, and of necessary belief function theory concepts. In Section 3, the BCP modeling of CVRPED is presented along with an analysis of how the optimal solution cost is influenced by the parameters involved in the belief-based constraints. Experiments on CVRPED instances built from wellknown CVRP instances, are reported in Section 4 , before concluding in Section 5.

\section{Background}

In this section, the CCP modeling of CVRPSD is first recalled, and then some necessary concepts of belief function theory are reviewed.

\subsection{CCP Modeling of CVRPSD}

The Capacitated Vehicle Routing Problem (CVRP) is an important variation of VRP where vehicles have identical capacities, and customers have indivisible deterministic demands. It can be formulated as follows:

$$
\text { Minimize } \sum_{i=1}^{n} \sum_{j=1}^{n} c_{i, j} \sum_{k=1}^{m} w_{i, j, k},
$$

where $n$ is the number of customers including the depot, $m$ the number of vehicles that are initially located at the depot, $c_{i, j}$ the cost for traveling between customers $x_{i}$ and $x_{j}$, and $w_{i, j, k}$ a binary variable that is equal to 1 if vehicle $k$ goes from $x_{i}$ to $x_{j}$ and serves them, and 0 if it does not. Besides, routes must be designed so that each route starts and ends at the depot and so that each customer is visited exactly once by exactly one vehicle; due to lack of space, we refer to [1] for a formal description of these constraints. In addition, the sum of the demands of the customers served by a route must not exceed vehicle capacity, which corresponds to the capacity constraints

$$
\sum_{i=1}^{n} d_{x_{i}} \sum_{j=1}^{n} w_{i, j, k} \leq Q, \quad k=1, \ldots, m,
$$


where $d_{x_{i}}$ is the quantity demanded by customer $x_{i}$ and $Q$ the vehicle capacity.

We are interested by a variation of CVRP, called CVRPSD, which introduces stochastic demands into CVRP, i.e., $d_{x_{i}}, i=1, \ldots, n$, are now random variables. A way to address this problem is via the CCP approach, which corresponds to the same optimization problem as CVRP except that constraints represented by (2) are replaced by the following so called chance-constraints:

$$
P\left(\sum_{i=1}^{n} d_{x_{i}} \sum_{j=1}^{n} w_{i, j, k} \leq Q\right) \geq 1-\beta, \quad k=1, \ldots, m,
$$

where $1-\beta$ is the minimum allowable probability that any route respects vehicle capacity and thus succeeds.

\subsection{Belief Function Theory}

Belief function theory was introduced in [14] as a theory of evidence. In this theory, uncertain knowledge about a variable $\omega$ taking its values in a domain $\Omega$, is represented by a Mass Function (MF) defined as a mapping $m: 2^{\Omega} \rightarrow$ $[0,1]$ verifying $\sum_{A \subseteq \Omega} m(A)=1$ and $m(\emptyset)=0$. The mass $m(A)$ represents the probability of knowing only that $\omega \in A$. Every $A \subseteq \Omega$ such that $m(A)>0$, is a focal element of $m$. A mass function is called Bayesian if its focal elements are singletons (in which case it is the usual probability mass function) and categorical if it has only one focal element. To be consistent with the stochastic case notation and terminology, we will write $m(\omega \in A)$ instead of $m(A)$, and a variable $\omega$ whose true value is known in the form of a MF will be called an evidential variable.

Equivalent representations of a MF $m$ are the belief and plausibility functions defined, respectively, as

$$
\begin{aligned}
B e l(\omega \in A) & =\sum_{B \subseteq A} m(\omega \in B), \quad \forall A \subseteq \Omega, \\
P l(\omega \in A) & =\sum_{B \cap A \neq \emptyset} m(\omega \in B), \quad \forall A \subseteq \Omega .
\end{aligned}
$$

The degree of belief $\operatorname{Bel}(\omega \in A)$ can be interpreted as the probability that the evidence about $\omega$ and represented by $m$, supports (implies) $\omega \in A$, whereas the degree of plausibility $P l(\omega \in A)$ is the probability that the evidence is consistent with $\omega \in A$. We have $\operatorname{Bel}(\omega \in A) \leq P l(\omega \in A)$, for all $A \subseteq \Omega$. Besides, if $m$ is Bayesian, then $\operatorname{Bel}(\omega \in A)=\operatorname{Pl}(\omega \in A)$, for all $A \subseteq \Omega$, and this function is a probability measure.

In this paper, belief function theory is used to model uncertain knowledge about customer demands, which we assume to be positive real numbers. Hence, we will be dealing with MF defined on $\Omega=\mathbb{R}^{+}$. The tools of belief function theory exposed above remain the same in such case, as long as the number of focal sets is finite [11, which will be the case in this study. Besides, focal sets of MF considered in this paper will all be intervals of positive real numbers. 
Let us finally recall the definition provided by Yager [17, of the addition of evidential variables, which will also be needed in the next section.

Definition 1. Let $[w]=[\underline{w}, \bar{w}]$ denote the closed interval of all reals $w$, such that $\underline{w} \leq w \leq \bar{w}$. Let $\sigma$ and $\tau$ be two independent evidential variables defined on $\mathbb{R}^{+}$, and having finite numbers of focal sets, which are intervals. Their addition is the evidential variable $\sigma+\tau$ with associated mass function

$$
m(\sigma+\tau \in[u])=\sum_{[s]+[t]=[u]} m(\sigma \in[s]) \cdot m(\tau \in[t]),
$$

where the addition of two intervals $[s]$ and $[t]$ is defined by $[s]+[t]=[\underline{s}+\underline{t}, \bar{s}+\bar{t}]$.

Remark 1. Let $\sigma$ and $\tau$ be the evidential variables in Definition 1, Let $\bar{\sigma}$ and $\bar{\tau}$ be two independent random variables with associated probability mass functions $p_{\bar{\sigma}}$ and $p_{\bar{\tau}}$ defined by $p_{\bar{\sigma}}(\bar{s})=m(\sigma \in[\underline{s}, \bar{s}])$ and $p_{\bar{\tau}}(\bar{t})=m(\tau \in[\underline{t}, \bar{t}])$ for any focal sets $[\underline{s}, \bar{s}]$ and $[\underline{t}, \bar{t}]$ of $\sigma$ and $\tau$, respectively. We will refer to $\bar{\sigma}$ and $\bar{\tau}$ as the upper probabilistic versions of $\sigma$ and $\tau$. It can easily be shown that we have $\operatorname{Bel}(\sigma+\tau \leq Q)=P(\bar{\sigma}+\bar{\tau} \leq Q)$, for any $Q \in \mathbb{R}^{+}$.

\section{BCP Modeling of CVRPED}

This section formalizes, and then studies, a means to handle the case where uncertainty on customer demands in the CVRP is represented by belief functions.

\subsection{Formalization}

Let us consider the case where customer demands are no longer deterministic or random, but evidential, i.e., $d_{x_{i}}, i=1, \ldots, n$, are now evidential variables. The associated problem is then called CVRPED as already introduced in Section 1. Following what has been done in [9] for the case of linear programs under uncertainty, we may generalize the CCP modeling of CVRPSD into a Belief-Constrained Programming (BCP) modeling of CVRPED, which amounts to keeping the same optimization problem as CVRP except that capacity constraints represented by (2) are replaced by the following belief-constraints:

$$
\begin{aligned}
& \operatorname{Bel}\left(\sum_{i=1}^{n} d_{x_{i}} \sum_{j=1}^{n} w_{i, j, k} \leq Q\right) \geq 1-\underline{\beta}, \quad k=1, \ldots, m, \\
& P l\left(\sum_{i=1}^{n} d_{x_{i}} \sum_{j=1}^{n} w_{i, j, k} \leq Q\right) \geq 1-\bar{\beta}, \quad k=1, \ldots, m,
\end{aligned}
$$

with $\beta \geq \bar{\beta}$ and where $1-\beta$ (resp. $1-\bar{\beta}$ ) is the minimum allowable degree of belief (resp. plausibility) that a vehicle capacity is respected on any route. Note that in order to evaluate the belief-constraints (4) and (5), the total demand on every route must be determined by summing all customers demands on that route, which is done using Definition 1 . 


\subsection{Particular Cases of the BCP Modeling of CVRPED}

It is interesting to remark that depending on the values chosen for $\beta$ and $\bar{\beta}$ as well as the nature of the evidential demands $d_{x_{i}}, i=1, \ldots, n$, the BCP modeling of CVRPED may degenerate into simpler or well-known optimisation problems.

The case $\beta=\bar{\beta}$ is particularly important. In this case, constraints (5) can be dropped, that is, only constraints (4) need to be evaluated (if constraints (4) are satisfied then constraints (5) are necessarily satisfied due to the relation between the belief and plausibility functions). As a matter of fact, the BCP approach originally introduced in [9] is of this form (no constraint based on $\mathrm{Pl}$ is considered). Furthermore, we note that due to Remark 1 , the BCP modeling of CVRPED can be converted into an equivalent optimisation problem, which is the CCP modeling of a CVRPSD where the stochastic demand of client $x_{i}$ is defined as the upper probabilistic version $\overline{d_{x_{i}}}$ of its evidential demand $d_{x_{i}}$. In particular, if the evidential demands are Bayesian, i.e., we are dealing really with a CVRPSD, then the BCP modeling clearly degenerates into the CCP modeling of this CVRPSD. In contrast, if the evidential demands are categorical, i.e., we are dealing with a CVRP where each customer demand $d_{x_{i}}$ is only known to belong to an interval $\left[d_{x_{i}}, \overline{d_{x_{i}}}\right]$, then the belief-constraints reduce to the following constraints

$$
\sum_{i=1}^{n} \overline{d_{x_{i}}} \sum_{j=1}^{n} w_{i, j, k} \leq Q, \quad k=1, \ldots, m,
$$

since in the case of categorical demands, the total demand on any given route is also categorical (it corresponds to the interval whose endpoints are obtained by summing the endpoints of the interval demands of the customers on the route) and thus for any $k=1, \ldots, m, \operatorname{Bel}\left(\sum_{i=1}^{n} d_{x_{i}} \sum_{j=1}^{n} w_{i, j, k} \leq Q\right)$ either

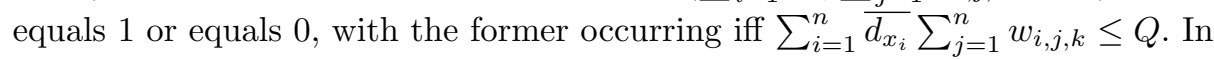
other words, in the case of categorical demands and $\beta=\bar{\beta}$, the BCP modeling amounts to searching the solution which minimizes the overall cost of servicing the customers (1) under constraints (6), i.e., assuming the maximum (worst) possible customer demands, and thus it corresponds to the minimax optimisation procedures encountered in robust optimization [16].

Let us eventually remark that the case $\beta=1>\bar{\beta}$ is the converse of the case $\beta=\bar{\beta}$ in the sense that constraints (4) can be dropped (as they are necessarily satisfied) and only constraints (5) need then to be evaluated. Moreover, as in the case $\beta=\bar{\beta}$, the BCP modeling of CVRPED can be converted into an equivalent optimisation problem, which is the CCP modeling of some CVRPSD (this is due to the existence of a counterpart to Remark 1 for the plausibility function, which relies on the lower endpoints of the focal sets rather than the upper endpoints).

\subsection{Influence of $\beta, \bar{\beta}$ and $Q$ on the Optimal Solution Cost}

In this section, we study the influence of the parameters $\beta, \bar{\beta}$ and $Q$ on the cost of the optimal solution of the CVRPED modeled via BCP. 
To simplify the presentation, we will denote by $\Sigma_{Q, \underline{\beta}, \bar{\beta}}$ the set of solutions to the CVRPED modeled via BCP and $\hat{C}_{Q, \underline{\beta}, \bar{\beta}}$ the cost of an optimal solution in $\Sigma_{Q, \beta, \bar{\beta}}$, for some $\underline{\beta}, \bar{\beta}$ and $Q$.

$\bar{T}$ he following propositions state how the optimal solution cost changes as $Q$, $\underline{\beta}$ or $\bar{\beta}$ vary.

Proposition 1. The optimal solution cost is non increasing in $Q$.

Proof. Let us consider a set $\mathcal{C}=\left\{R_{1}, \ldots, R_{m}\right\}$ composed of $m$ routes $R_{k}, k=$ $1, \ldots, m$, such that it is not known whether this set respects the belief-constraints (4) and (5), but it is known that it respects all the other constraints of the CVRPED modeled via BCP, in particular each route $R_{k}$ starts and ends at the depot and each customer is visited exactly once by exactly one vehicle.

It is clear that for any $\beta$ and $\bar{\beta}$, as $Q$ increases (starting from 0 ), it reaches necessarily a value at which $\mathcal{C}$ becomes a solution to the CVRPED modeled via BCP. Hence, $\Sigma_{Q, \underline{\beta}, \bar{\beta}} \subseteq \Sigma_{Q^{\prime}, \underline{\beta}, \bar{\beta}}$ for $Q^{\prime} \geq Q$, and thus $\hat{C}_{Q^{\prime}, \underline{\beta}, \bar{\beta}} \leq \hat{C}_{Q, \underline{\beta}, \bar{\beta}}$.

Proposition 2. The optimal solution cost is non increasing in $\underline{\beta}$.

Proof. Let us consider a set $\mathcal{C}=\left\{R_{1}, \ldots, R_{m}\right\}$ composed of $m$ routes $R_{k}, k=$ $1, \ldots, m$, such that it is not known whether this set respects the belief-constraints (4), but it is known that it respects all the other constraints of the CVRPED modeled via BCP, in particular constraints (5).

It is clear that for any $Q$, as $\beta$ increases from $\bar{\beta}$ to 1 , it reaches necessarily a value at which $\mathcal{C}$ becomes a solution to the CVRPED modeled via BCP. Hence, $\Sigma_{Q, \underline{\beta}, \bar{\beta}} \subseteq \Sigma_{Q, \underline{\beta}^{\prime}, \bar{\beta}}$ for $\underline{\beta}^{\prime} \geq \underline{\beta}$, and thus $\hat{C}_{Q, \underline{\beta}^{\prime}, \bar{\beta}} \leq \hat{C}_{Q, \underline{\beta}, \bar{\beta}}$.

Proposition 3. The optimal solution cost is non increasing in $\bar{\beta}$.

Proof. The proof is similar to that of Proposition 2

Informally, Propositions 13 show that if a decision maker is willing to buy vehicles with a higher capacity or to have vehicle capacity exceeded on any route more often, then he will obtain at least as good (at most as costly) solutions in theory, i.e., if he uses an exact optimization method. Unfortunately, no such method exists yet for the CVRPED modeled via BCP. As a matter of fact, the next section reports an experiment, where solutions to this optimization problem are sought using a metaheuristic.

\section{Experimental Study}

Section 3 has introduced the CVRPED modeled via BCP, and has studied some of its theoretical properties. In this section, a preliminary experimental study on some CVRPED instances is presented for the case where $\underline{\beta}=\bar{\beta}$; for notation simplicity we introduce the value $\beta$ such that $\beta=\beta=\bar{\beta}$. These instances are first described, and then the obtained results are discussed. Note that to solve these 
instances, we adapted a simulated annealing algorithm designed for the CVRP, which was proposed in [5] and that uses a combination of random and greedy operators based on problem knowledge. However, due to space limitations, we must refrain from describing this adaptation.

\subsection{CVRPED Instances}

We have generated CVRPED instances based on Augerat set A instances for the CVRP [13]. In our instances, the customers coordinates and the capacity constraints are the same as in Augerat's. However, each deterministic customer demand $d^{\text {det }}$ in Augerat instances has been replaced by an evidential demand $d^{e v}$ with associated MF

$$
\begin{aligned}
m\left(d^{e v} \in\left[d^{d e t}, d^{d e t}\right]\right) & =\alpha, \\
m\left(d^{e v} \in\left[d^{d e t}-\gamma \cdot d^{d e t}, d^{d e t}+\gamma \cdot d^{d e t}\right]\right) & =1-\alpha,
\end{aligned}
$$

with $\alpha \in[0,1]$ and $\gamma \in(0,1)$. Such a transformation of the original deterministic demand may be relevant if each customer providing his deterministic demand is assumed to be reliable with probability $\alpha$, and approximately (at $\pm \gamma * 100 \%$ ) reliable with probability $1-\alpha[12$.

We note that a deterministic demand is a particular case of an evidential demand, and thus the BCP approach to the CVRPED can also be applied to CVRP instances. Although this latter idea may not be very useful in itself, it leads to an interesting remark based on the following result.

Proposition 4. For any $\beta$ and $Q$, the optimal solution to a CVRPED instance generated from a CVRP instance through transformation (7) and modeled via the BCP approach, has a higher or equal cost to that of the optimal solution of the CVRP instance modeled via the BCP approach.

Proof. Let $\Sigma_{Q, \beta, \beta}^{e v}$ and $\Sigma_{Q, \beta, \beta}^{\text {det }}$ be the sets of solutions to the CVRPED and CVRP instances modeled via BCP, for some $Q$ and $\beta$. For any route $R_{k}$ of any solution $S \in \Sigma_{Q, \beta, \beta}^{e v}$, it can easily be shown that we have $\operatorname{Bel}\left(d_{R_{k}}^{e v} \leq Q\right) \leq$ $\operatorname{Bel}\left(d_{R_{k}}^{\text {det }} \leq Q\right)$, where $d_{R_{k}}^{e v}$ and $d_{R_{k}}^{\text {det }}$ are evidential variables denoting respectively the sum of the evidential and deterministic demands on $R_{k}$, and thus any solution $S \in \Sigma_{Q, \beta, \beta}^{e v}$ also belongs to $\Sigma_{Q, \beta, \beta}^{d e t}$. We have thus $\Sigma_{Q, \beta, \beta}^{e v} \subseteq \Sigma_{Q, \beta, \beta}^{\text {det }}$.

The cost difference put forward by Proposition 4 between the optimal solution(s) of a CVRPED instance generated using (7) and the optimal solution(s) of its original generating CVRP instance, represents what a decision maker would loose if the customers were actually totally reliable whereas he was cautious and thought (wrongly) that they were only partially reliable. More specifically, by using (7), the decision maker assumes that the customer demands may be actually higher than they appear, i.e., he believes that the customers may underestimate their needs, and the price to pay by being cautious in that latter way, is that the optimal solution he will obtain may have a higher cost than if he had not made such an assumption. 


\subsection{Results Using Simulated Annealing}

We have generated CVRPED instances using the procedure described in the preceding section, where $\alpha$ was set to 0.8 , while $\gamma$ was set to 0.1 . We also chose $\beta=0.1$. The running time for the algorithm was less than an hour in almost all cases. Each instance was solved 30 times, and the results are given in Table 1 .

The column "Best cost CVRP" gives the costs of the best solutions reported so far for the CVRP instances [13. We note that for $\beta \neq 1$, the optimal solutions of the CVRP instances modeled via the BCP approach are the same as the optimal solutions of the CVRP instances, hence the costs in the column "Best cost CVRP" may be taken as the costs of the best solutions for the CVRP instances modeled via the $\mathrm{BCP}$ approach.

The "Difference with CVRP" column shows that for all instances the cost of the best solution for the CVRP is better than that of the best solution obtained for the CVRPED. This latter observation may be seen as an approximation of the theoretical difference between the optimal solution cost of a CVRP instance and the optimal solution cost of the CVRPED instance generated from it, which is predicted by Proposition 4 however, one must be careful since it is not possible to quantify the quality of this approximation due to the diversity and complexity of the algorithms involved in computing those values.

\section{Conclusions}

This paper studied the capacitated vehicle routing problem with evidential demands. We modeled this problem using a belief function based extension of the chance-constrained programming approach to stochastic mathematical programs. Furthermore, theoretical results relating variations of the optimal solution cost with variations of the parameters involved in the model, were provided. Instances of this difficult optimization problem were also solved using a metaheuristic. Future works includes addressing this problem using a recourse-based approach, which is another main approach to modeling stochastic mathematical programs [3]. Another perspective is to identify the customers whom more knowledge about their demands would lead to better solutions, that is performing a sensitivity analysis [4].

\section{References}

1. L. D. Bodin, B. L. Golden, A. A. Assad, and M. O. Ball. Routing and scheduling of vehicles and crews: The state of the art. Comput Oper Res, 10(2):63-212, 1983.

2. A. Charnes and W. W. Cooper. Chance-constrained programming. Manag Sci, 6(1):73-79, 1959.

3. J.-F. Cordeau, G. Laporte, M. W. P. Savelsbergh, and D. Vigo. Vehicle routing. In C. Barnhart and G. Laporte, editors, Handbooks in Oper Res Manag Sci, volume 14, chapter 6, pages 367-428. Elsevier, 2007.

4. S. Ferson and W. T. Tucker. Sensitivity in risk analyses with uncertain numbers. Technical report, Sandia National Laboratories, 2006. 
Table 1. Results of the simulated annealing algorithm on the CVRPED instances

\begin{tabular}{|l|c|c|c|c|c|c|c|}
\hline Instance & $\begin{array}{c}\text { Best } \\
\text { cost } \\
\text { CVRP }\end{array}$ & $\begin{array}{c}\text { Best } \\
\text { CVRt }\end{array}$ & $\begin{array}{c}\text { Worst } \\
\text { Cost } \\
\text { CVRPED }\end{array}$ & $\begin{array}{c}\text { Avg } \\
\text { cost }\end{array}$ & $\begin{array}{c}\text { Stand. } \\
\text { CVRPED } \\
\text { CVRPED }\end{array}$ & $\begin{array}{c}\text { Difference } \\
\text { with } \\
\text { CVRP }\end{array}$ & $\begin{array}{c}\text { Avg runtime } \\
\text { CVRPED } \\
\text { (seconds) }\end{array}$ \\
\hline A-n32-k5 & 784 & 802,07 & 837,04 & 822,27 & 8,3 & $2.3 \%$ & 1919,79 \\
\hline A-n33-k5 & 661 & 690,41 & 706,36 & 696,33 & 3,9 & $4.45 \%$ & 2147,13 \\
\hline A-n33-k6 & 742 & 777,63 & 782,80 & 779,84 & 1,4 & $4.8 \%$ & 2572,64 \\
\hline A-n34-k5 & 778 & 800,96 & 809,94 & 802,67 & 2,8 & $2.95 \%$ & 2727,92 \\
\hline A-n36-k5 & 799 & 849,83 & 887,47 & 866,67 & 9,02 & $6.36 \%$ & 2383,9 \\
\hline A-n37-k5 & 669 & 692,34 & 740,17 & 715,85 & 14,54 & $3.49 \%$ & 2107,05 \\
\hline A-n37-k6 & 949 & 1006,39 & 1054,31 & 1027,04 & 12,4 & $6.05 \%$ & 2131,87 \\
\hline A-n38-k5 & 730 & 770,34 & 819,54 & 796,9 & 12,4 & $5.53 \%$ & 2083,72 \\
\hline A-n39-k5 & 822 & 876,47 & 934,18 & 909,61 & 17,18 & $6.63 \%$ & 2109,3 \\
\hline A-n39-k6 & 831 & 855,18 & 895,45 & 874,7 & 8,8 & $2.9 \%$ & 2479,13 \\
\hline A-n44-k6 & 937 & 998,16 & 1108,10 & 1056,44 & 30,5 & $6.53 \%$ & 2611,04 \\
\hline A-n45-k6 & 944 & 1017,29 & 1083,19 & 1055,9 & 17,12 & $7.76 \%$ & 2276,26 \\
\hline A-n45-k7 & 1146 & 1194,19 & 1237,3 & 1212,23 & 12,08 & $4.2 \%$ & 2917,09 \\
\hline A-n46-k7 & 914 & 1014,87 & 1060,3 & 1034,81 & 11,02 & $11.03 \%$ & 2314,7 \\
\hline A-n48-k7 & 1073 & 1150,32 & 1201,80 & 1174,61 & 14,7 & $7.2 \%$ & 2742,24 \\
\hline A-n53-k7 & 1010 & 1145,46 & 1233,65 & 1181,39 & 18,9 & $13.4 \%$ & 2555,31 \\
\hline A-n54-k7 & 1167 & 1297,98 & 1409,6 & 1336,05 & 25,5 & $11.22 \%$ & 2530,67 \\
\hline A-n61-k9 & 1034 & 1121,58 & 1162,52 & 1142,31 & 11,5 & $8.47 \%$ & 3183,39 \\
\hline A-n62-k8 & 1288 & 1392,68 & 1448,29 & 1414,21 & 14,3 & $8.13 \%$ & 3990,58 \\
\hline A-n63-k9 & 1616 & 1835,86 & 1959,45 & 1890,48 & 30,8 & $13.61 \%$ & 2948,24 \\
\hline A-n65-k9 & 1174 & 1326,63 & 1400,09 & 1366,96 & 18,97 & $13 \%$ & 3097,57 \\
\hline A-n80-k10 & 1763 & 2098,53 & 2284,5 & 2191,77 & 48,3 & $19.03 \%$ & 3459,88 \\
\hline
\end{tabular}


5. H. Harmanani, D. Azar, N. Helal, and W. Keirouz. A simulated annealing algorithm for the capacitated vehicle routing problem. In 26th Int Conf on Computers and their Applications, New Orleans, USA, 2011.

6. M. J. L. Kirby. The current state of chance-constrained programming. In H. W. Kuhn, editor, Proc of the Princeton Symp on Mathematical Programming, pages 93-111. Princeton University Press, 1970.

7. S. Kirkpatrick, C. D. Gelatt, and M. P. Vecchi. Optimisation by simulated annealing. Science, 220(4598):671-680, 1983.

8. G. Laporte, F. V. Louveaux, and L. van Hamme. An integer l-shaped algorithm for the capacitated vehicle routing problem with stochastic demands. Oper Res, 50:415-423, 2002.

9. H. Masri and F. Ben Abdelaziz. Belief linear programming. Int J Approx Reason, 51:973-983, 2010.

10. Z. P. Mourelatos and J. Zhou. A design optimization method using evidence theory. J Mech Design, 128:901-908, 2006.

11. G. Nassreddine, F. Abdallah, and T. Denoeux. State estimation using interval analysis and belief function theory: Application to dynamic vehicle localization. IEEE Trans Syst Man Cybern B, 40(5):1205-1218, 2010.

12. F. Pichon, D. Dubois, and T. Denoeux. Relevance and truthfulness in information correction and fusion. Int J Approx Reason, 53(2):159-175, 2012.

13. Vehicle Routing Data Sets. http://www.coin-or.org/SYMPHONY/branchandcut/ VRP/data/index.htm Accessed: 2016-03-20.

14. G. Shafer. A mathematical theory of evidence. Princeton University Press, 1976.

15. R. K. Srivastava, K. Deb, and R. Tulshyan. An evolutionary algorithm based approach to design optimization using evidence theory. J Mech Design, 135(8), 2013.

16. I. Sungur, F. Ordónez, and M. Dessouky. A robust optimization approach for the capacitated vehicle routing problem with demand uncertainty. IIE Trans, 40:509523, 2008 .

17. R. R. Yager. Arithmetic and other operations on Dempster-Shafer structures. Int J Man Mach Stud, 25(4):357-366, 1986. 